\title{
Hommage à Pierre Guibbert
}

\section{Pierre Boutan}

\section{(2) OpenEdition}

Journals

Édition électronique

URL : http://journals.openedition.org/trema/566

DOI : $10.4000 /$ trema.566

ISSN : 2107-0997

Éditeur

Faculté d'Éducation de l'université de Montpellier

\section{Édition imprimée}

Date de publication : 1 mai 2004

Pagination : 1-2

ISSN : 1167-315X

\section{Référence électronique}

Pierre Boutan, « Hommage à Pierre Guibbert », Tréma [En ligne], 23 | 2004, mis en ligne le 05 janvier 2010, consulté le 22 septembre 2020. URL : http://journals.openedition.org/trema/566 ; DOI : https:// doi.org/10.4000/trema.566

Ce document a été généré automatiquement le 22 septembre 2020

Trema 


\title{
Hommage à Pierre Guibbert
}

\author{
Pierre Boutan
}

1 Pierre Guibert a succombé à une brutale crise cardiaque, dans les premières heures du 29 janvier 2004. Il avait mis un terme à sa carrière de maître de conférence à l'IUFM de Montpellier, où il dirigeait le Centre d'études, de documentation et de recherche en histoire de l'éducation (CEDRHE). Il était aussi directeur de recherches à l'université Paul VALERY, au titre de l'équipe Didaxis - Dipralang de Sciences du langage. Il venait donc tout juste d'entamer une retraite active, tant dans le domaine du cinéma que comme nouveau président des Amis de la mémoire pédagogique de l'Hérault.

2 La revue Tréma lui doit beaucoup: non seulement par les articles qu'il y a publié (du numéro $1:$ : Le mythe de la Commune dans les manuels de la première histoire ", au numéro 14 où il présente le Centre), mais aussi par le choix des illustrations et le travail obscur mais indispensable de relecture des numéro jusqu'en 2000 .

3 Fils d'un couple d'instituteurs du Biterrois, il effectue des études classiques au lycée de Béziers puis en hypokhâgne au lycée JOFFRE de Montpellier. Après sa licence de lettres modernes à l'université Paul VALERY, il est professeur dans divers établissements secondaires de l'Hérault puis à Bram dans l'Aude, enfin au lycée MERMOZ de 
Montpellier, où il enseigne, de manière expérimentale alors, le cinéma en section tourisme. Il vient d'ailleurs de passer une volumineuse thèse sur le metteur en scène soviétique Eisenstein. C'est alors qu'il rencontre Marcel OMS, avec lequel il travaille au Cahiers de la cinémathèque, à l'institut Jean VIGO et au festival «Confrontation" de Perpignan.

En 1975 commence sa carrière de formateur de maîtres, lorsqu'il est nommé à l'école normale d'institutrices de Montpellier. En tant que nouveau formateur, il effectue son stage d'adaptation d'un an à l'EN des Batignolles à Paris ... Il y fait la connaissance de Claude BILLARD, avec lequel il publiera un ouvrage remarqué - en première page du Monde - L'Histoire mythologique des Français. C'est un travail d'analyse de manuels scolaires: sa seconde passion. Suit Le petit catéchisme illustré ou la fin d'un mythe. Il devient le collaborateur régulier des Lundis de l'Histoire de Denis Richet sur France Culture. Les amitiés franco - allemandes le voient aussi intervenir souvent de l'autre côté du Rhin, en particulier dans la revue GEORG - ECKERT - Institut de Braunschweig, Internationale Schulbuchforschung.

5 Au début des années 80, avec Michel VERDELHAN et Carmen TANCHON, il publie une collection de manuels de français pour le primaire.

Il se lie par ailleurs avec Guy HENNEBELLE, avec lequel il contribue à la publication des revues Ciném'action et Panoramiques.

7 A partir de sa propre collection, il constitue en 1992 à l'IUFM de Montpellier un fonds unique de manuels et d'ouvrages de pédagogie, de films fixes, de cahiers, d'images anciennes ... et il passe un temps considérable à un inventaire de ses richesses sur bases de données (plus de 30000 documents), travail alors sans équivalent en France (aujourd'hui le CEDRHE).

Il venait de publier L'école de la Revanche (1870-1919) et mettait la dernière main à une exposition et un ouvrage sur "L'école, la vigne et le vin", pour les Amis de la Mémoire pédagogique, avec la collaboration du CEDRHE, exposition qu'il ne verra pas.

9 Le comité de rédaction de la revue Tréma avec la direction de l'IUFM de l'académie de Montpellier, ses collègues, ses amis, transmettent à sa famille leur témoignage de profonde sympathie. 